\title{
Die Bedeutung der IV für psychisch Kranke
}

\section{Daniel Hell}

Prof. Dr. med., Klinikdirektor Psychiatrische Universitätsklinik Zürich
Korrespondenz:

Prof. Dr. med. Daniel Hell Psychiatrische Universitätsklinik Zürich

Lenggstrasse 31

Postfach 1931

CH-8032 Zürich

Tel. 0443842312

Fax 0443842719

hellsek@bli.unizh.ch
Wenn heute gefordert wird, dass aus der Rentenversicherung eine Eingliederungsversicherung werden soll, so ist daran zu erinnern, dass erst die Einführung der IV-Renten die soziale Integration schwerer psychisch Kranker ermöglicht hat. Bevor es in der Schweiz eine Invalidenversicherung gab, waren diese Kranken oft über Jahre und Jahrzehnte in psychiatrischen Kliniken, die eine Asylfunktion hatten, untergebracht. Erst mit Einführung der IV im Jahre 1960 bekamen sozialpsychiatrische Integrationsbemühungen eine Realisierungschance. Es waren neben beruflichen Wiedereinstiegshilfen («Arbeit vor Rente») vor allem die heute gerne beargwöhnten Renten, die zum Exodus chronisch psychisch Kranker aus psychiatrischen Anstalten Anlass gaben. Erst die IV-Renten ermöglichten, dass früher asylierte Kranke in Wohnheime und betreute Wohnungen überwechseln und in geschützten Werkstätten und Ateliers arbeiten konnten. Ohne diese finanzielle Hilfe wäre es trotz verbesserter pharmakologischer und psychotherapeutischer Behandlung nicht möglich gewesen, Alternativen zur stationären Unterbringung aufzubauen und die psychisch kranken Menschen vermehrt in die Gemeinden zu integrieren.

\section{Renten können integrativ wirken}

Man darf sich nicht täuschen lassen. Die schwerer psychisch Kranken werden in Zukunft nicht weniger auf IV-Renten angewiesen sein als in den letzten Jahrzehnten. Der Abbau der Psychiatriebetten macht die finanziell unterstützte soziale Integration sogar noch notwendiger als früher, soll eine Verelendung grösseren Ausmasses verhindert werden. Es geht also nicht an, die Berentung gegen berufliche Integrationsbemühungen auszuspielen. Beides tut not, und beides hat einen Integrationseffekt.

Leider haben die diskriminierenden Äusserungen über psychisch Behinderte in den letzten Jahren und Monaten zusammen mit Sparbemühungen bereits dazu beigetragen, dass der Druck einzelner IV-Stellen auf psychisch Kranke erhöht wird. Statt die soziale oder berufliche Integration damit zu fördern, wird oft das Gegenteil erreicht: Die Betroffenen werden hilflos gemacht und fühlen sich unter Druck überfordert.

Was für die Arbeitgeberseite recht ist, nämlich mit «Engagement und Sensibilität, aber

\section{Portée de l'Al pour \\ les malades psychiques}

Si I'on exige aujourd'hui que l'on crée une assurance de réadaptation en lieu et place de l'assurance de la rente, il convient de rappeler que seule I'introduction de rentes $\mathrm{Al}$ a permis l'intégration sociale de malades psychiques graves. Les patients gravement atteints dans leur psychisme ne seront pas moins dépendants des rentes $\mathrm{Al}$ que ces dernières décennies. La diminution des lits en psychiatrie a pour conséquence de rendre encore plus nécessaire la rémunération financière de l'intégration sociale, si l'on veut éviter une plus grande paupérisation. II ne s'agit donc pas de mettre en opposition la rente contre les efforts d'intégration professionnelle. Les deux sont indispensables et ont un effet d'intégration.

II ne sert pas à grand-chose non plus de faire subir une pression existentielle aux malades psychiques et de les replacer dans les mêmes conditions de travail dont ils ont été victimes. Il s'agit bien davantage de soulager et d'intégrer - p.ex. d'octroyer des rentes partielles et de prendre des mesures de réintégration professionnelle - afin d'assurer le plus grand nombre possible de places de travail à des handicapés (psychiques).

nicht (mit) mehr Vorschriften und Zwang» (A. Schuppisser vom Arbeitgeberverband) behandelt zu werden, sollte in einem liberalen Rechtsstaat auch für Behinderte gelten. Bei Annahme der 5. IV-Revision wird von krankheitsbedingt arbeitsunfähigen Menschen «bedingungslose Mitwirkung» gefordert. Gleichzeitig soll es aber möglich sein, dass sie «ohne ihre Mitwirkung», d.h., ohne dass sie überhaupt gefragt werden, von Arbeitgebern, Ärzten, Angehörigen und anderen Personen bei der IV (nach 4 Wochen Arbeitsunfähigkeit) angemeldet werden. Dieses Missverhältnis ist mit liberalem Gedankengut nicht zu vereinbaren. Ein solches Vorgehen hat auch 
zur Konsequenz, dass viele erkrankte oder behinderte Menschen sich ungerecht behandelt fühlen. Das Gefühl, benachteiligt zu sein, ist aber eine denkbar schlechte Voraussetzung zur Integration.

\section{Vertrauensbildung statt Druck}

Der heutige Wissensstand über berufliche Integrationsbemühungen psychisch Behinderter lässt zudem den Schluss zu, dass eine Reintegration in den Arbeitsprozess ohne gegenseitiges Vertrauen - also ohne Vertrauensbildung zwischen Arbeitgeber, behindertem Arbeitnehmer und Coach - unmöglich ist. Druck und Drohungen - etwa die IV-Leistungen zu kürzen, wenn den Vorschlägen der IV-Stelle nicht Folge geleistet wird - werden voraussichtlich die Integrationsbemühungen hemmen statt fördern.

Auch die guten Erfahrungen an der Psychiatrischen Universitätsklinik Zürich mit der freiwilligen Unterstützung von psychiatrischen Patienten und Arbeitgebern zur beruflichen Wiedereingliederung (sogenanntes «supported employment») weisen darauf hin, dass eine Integration geduldig und ohne Zeitdruck zu erfolgen hat, soll sie erfolgreich sein. Überlastungen führen bei psychisch Kranken oft zu emotio- nalen Schwankungen, die aber mit geduldiger Unterstützung überwunden werden können. Es ist deshalb unrealistisch, von psychisch verletzten Menschen zu erwarten, dass sie imstande sind, zeitlich stark befristete Integrationsmassnahmen zu nutzen. Viele heutige psychisch Kranke fühlen sich als Opfer des gestiegenen Arbeitsdruckes in der Wirtschaft. Viele haben Routinearbeiten oder Nischenarbeitsplätze verloren, die wegrationalisiert oder in Billiglohnländer verlagert wurden. Die tiefgehende Umstrukturierung der Arbeitswelt im letzten Jahrzehnt kann - selbst bei günstigsten wirtschaftlichen Bedingungen - nicht damit wettgemacht werden, dass man über sie hinwegsieht. Wie Blinde trotz ihrer Bemühungen wiederholt an der Visualisierung der modernen Arbeitswelt scheitern, kommen psychisch Kranke häufig mit der Forderung nach Teamfähigkeit, Flexibilität und Mobilität nicht zurecht. Es nützt wenig, psychisch Kranke unter Existenzdruck zu setzen und wieder den gleichen Arbeitsbedingungen zu unterwerfen, deren Opfer sie wurden. Vielmehr braucht es Entlastung und Integration - z.B. Teilrente und berufliche Massnahmen -, um möglichst viele Arbeitsplätze für (psychisch) Behinderte zu sichern. 